\title{
Subjective, Autonomic, and Endocrine Reactivity during Social Stress in Children with Social Phobia
}

\author{
Martina Krämer • Wiebke Lina Seefeldt • \\ Nina Heinrichs • Brunna Tuschen-Caffier • \\ Julian Schmitz • Oliver Tobias Wolf • Jens Blechert
}

(C) Springer Science+Business Media, LLC 2011

\begin{abstract}
Reports of exaggerated anxiety and physiological hyperreactivity to social-evaluative situations are characteristic of childhood social phobia (SP). However, laboratory research on subjective, autonomic and endocrine functioning in childhood SP is scarce, inconsistent and limited by small sample sizes, limited breadth of measurements, and the use of non-standardized stressor tasks. We exposed 812-year-old children with DSM-IV SP $(n=41)$ and matched healthy control children (HC; $n=40)$ to the Trier Social Stress Test for Children (TSST-C) while measuring subjective anxiety, heart rate (HR) and salivary alpha-amylase (sAA) as well as salivary cortisol. The SP children showed heightened reactivity to the TSST-C on subjective anxiety compared to the $\mathrm{HC}$ children but not a heightened reactivity
\end{abstract}

\footnotetext{
M. Krämer $(\bowtie) \cdot$ B. Tuschen-Caffier · J. Schmitz

Department of Clinical Psychology and Psychotherapy,

University of Freiburg,

Engelbergerstrasse 41,

79106 Freiburg, Germany

e-mail: martina.kraemer@psychologie.uni-freiburg.de
}

W. L. Seefeldt

Department of Clinical Psychology, Psychotherapy

and Diagnostic, Technical University of Braunschweig,

Braunschweig, Germany

\section{N. Heinrichs}

Department of Clinical Child and Adolescent

Psychology and Psychotherapy, University of Bielefeld,

Bielefeld, Germany

\section{O. T. Wolf}

Department of Cognitive Psychology, University of Bochum,

Bochum, Germany

\section{J. Blechert}

Department of Psychology, University of Salzburg,

Salzburg, Austria in HR, sAA or cortisol. However, the SP children showed chronically elevated HR levels throughout the whole laboratory session. Whereas subjective anxiety seems to respond specifically to social-evaluative stress in childhood SP, HR levels may be chronically elevated suggesting a more generalized autonomic hyperreactivity.

Keywords Social phobia · Children · Social stress · Physiological reactivity. Heart rate $\cdot$ Cortisol

\section{Introduction}

Social phobia (SP) is one of the most common mental disorders in adults, adolescents and children with lifetime prevalence rates of up to $12,1 \%$ (Ruscio et al. 2008). In the Diagnostic Statistical Manual of Mental Disorders (DSMIV; American Psychiatric Association [APA], 1994), SP is defined as an excessive, irrational fear of social or performance situations due to the expectation that others will scrutinize the person's actions. The average age of onset for SP is early to mid-adolescence but SP occurs in children as young as 8 years (Beidel et al. 1999). SP shows high comorbidity rates, is associated with a significant impairment of social functioning and often follows a chronic course (Kessler 2003; Schneier et al. 1992). Because of its early onset, SP can lead to an impaired social and emotional development (Beidel et al. 1999). However, there is still limited literature about the psychopathology of SP in children (Beidel et al. 1999; Rao et al. 2007; Spence et al. 1999). It remains unclear whether the results from adult and adolescent research apply to children whose biological, cognitive and emotional systems still underlie developmental processes (Ollendick and HirshfeldBecker 2002; Rao et al. 2007). A better understanding of 
the causes and correlates of childhood SP is essential to develop and improve treatment for affected children.

Physiological anxiety symptoms in current nosology and models of SP

In current conceptualizations of SP in adulthood, physiological arousal plays an important role. Panic-like symptoms with various physiological arousal symptoms are part of the DSM-IV diagnosis of SP. Physiological hyperarousal symptoms also figure prominently in cognitive models of SP (e.g. Clark and Wells 1995; Rapee and Heimberg 1997), which identify the attention to, and misinterpretation of internal physiological arousal as one key factor in the maintenance of the disorder. These models propose that individuals with SP infer from physiological sensations how they appear to others: when socially phobic individuals perceive anxiety symptoms such as blushing, sweating or an elevated heart rate, they will become more anxious because others might see evidence of their nervousness. An interesting question here is whether individuals with SP are really abnormally aroused or if their strongly perceived arousal is merely an effect of excessive self-focused attention. If the latter is true, heightened physiological arousal may not be a necessary condition for the manifestation of pathological social anxiety.

Symptom provocation and the multiple response levels of anxiety

To investigate if individuals with SP actually do show heightened physiological arousal in social situations, a first step in experimental psychopathology has frequently been symptom provocation - the elicitation of symptoms under controlled laboratory conditions. In addition to self-report measures, which measure the experience of anxiety, stress and anxiety can be assessed at many different response levels.

In the context of social-evaluative stress and anxiety two physiological response systems have been studied intensively: The hypothalamus-pituary-adrenal (HPA) system and the autonomic nervous system (ANS). In stress situations, the hypothalamic production of corticotropinreleasing hormone (CRF) rises, which stimulates the pituary release of adrenocorticotropin hormone (ACTH) and, as a consequence, cortisol is secreted into the bloodstream by the adrenal cortex (Axelrod and Reisine 1984). Cortisol can be assessed in the saliva and is therefore a popular non-invasive index of HPA-axis activity. The ANS consists of a sympathetic and a parasympathetic branch and allows for fast adaptations of a large number effector organs. One of the most common indicators of ANS activity is heart rate, reflecting the joint influences of both ANS branches (Berntson et al. 1994). However, the ANS also innervates the salivary glands and it has been found that the salivary enzyme Alpha-Amylase (sAA) reflects mainly sympathetic ANS activity (Nater and Rohleder 2009; Nater et al. 2005). Thus, the parallel assessment of HR, SAA, and cortisol together with subjective anxiety should adequately reflect the two major physiological systems implicated in the human response to social stress.

Research on social-evaluative stress in adults, adolescents, and children with SP

Previous research in adults and adolescents with SP has provided an inconsistent pattern of results regarding the question of whether social anxiety might be characterized by elevated subjective, autonomic and endocrine reactivity to social-evaluative stress. According to some studies, SP individuals exposed to a social-evaluative task respond with increased subjective anxiety and an increased HR response (Hofmann et al. 1995) as well as with elevated cortisol reactivity (Roelofs et al. 2009). Other studies, while replicating increased subjective anxiety in socially phobic or highly socially anxious individuals relative to controls, did not find differential cortisol or cardiovascular reactivity (Anderson and Hope 2009; Beaton et al. 2006; Grossman et al. 2001; Heiser et al. 2009; Mauss et al. 2003). The only study that investigated sAA in adults with SP and controls found elevated sAA levels in SP individuals, both in nonstressed conditions during the day and after a low dose $(0.5 \mathrm{mg})$ of dexamethasone. The authors conclude that these results suggest a hyperactivity of the sympathetic branch of ANS during basal, non-stimulated conditions in individuals with SP (van Veen et al. 2008).

Only a small number of studies has examined subjective and physiological reactivity to social stress in children with SP. Beidel (1991) found increased subjective anxiety reactivity to a read-aloud task in SP children compared to healthy controls (HC) but no differences in HR. Regarding cortisol reactivity, van West et al. (2008) examined 25 children with SP between 6 and 12 years of age and 25 control children during an adapted version of the Trier Social Stress Test (TSST; Kirschbaum et al. 1993), consisting of a 5-minute speech in front of an audience. The authors found higher anxiety levels as well as an elevated salivary cortisol response to the task in children with SP compared to HC. No autonomic measures were assessed.

\section{The present study}

In sum, it remains unclear if SP is characterized by autonomic and endocrine hyperreactivity to stress or whether its core symptomatology is to be found at the 
cognitive-experiential level. The studies reviewed above vary in statistical power, sample characteristics, breadth of measurements and stressor tasks, which precludes firm conclusions. In an attempt to address some of the limitations of previous studies, we conducted a wellpowered, multi-measure study of SP children and matched controls, who we exposed to a widely used and standardized social stressor, the TSST for Children (TSST-C; BuskeKirschbaum et al. 1997). The TSST-C includes two socialevaluative tasks (finishing a story and serial subtraction) to be completed in front of two unknown observers.

Our goal was to determine if SP children differ from HC children not only in terms of subjective anxiety but also on autonomic and endocrine stress measures. We expected elevated subjective anxiety in SP children (Beidel 1991; van West et al. 2008). No predictions were made for autonomic responses (HR, sAA) due to the heterogeneity of previous findings. Endocrine (salivary cortisol) responses were expected to be higher in children with SP compared to HC children (van West et al. 2008).

\section{Method}

\section{Participants}

The present study was part of a two-site research project consisting of a three-session study series on subjective, autonomic, and endocrine functioning of children with SP. Comparability across centers was achieved by using identical hard- and software for data acquisition and analysis, as well as identical tasks and interviews (assured through the exchange of videotaped sessions). The study was approved by the ethics committee of the German Society of Psychology. The current sample consisted of 41 children aged 8 to 12 years who met a primary DSM-IV diagnosis of SP, and $40 \mathrm{HC}$ children, matched by age and gender, who did not meet diagnostic criteria for any lifetime Axis I disorder. ${ }^{1}$ All children were Caucasian.

The SP and HC children were recruited through advertisements in local newspapers as well as psychological and medical treatment facilities for children. The children were offered vouchers worth $€ 25$ (approximately US\$33) in exchange for participation. Parents (who completed a structured interview and several questionnaires) received a reimbursement of $€ 25$.

At each study site, the Diagnostic Interview for Mental Disorders for Children (Kinder-DIPS; Schneider et al. 2009) was used to determine if the children met criteria for SP and if comorbid diagnoses were existent. The

\footnotetext{
${ }^{1}$ The current sample is a sub-sample of the whole sample $(n=130)$ for which endocrine data were available.
}

Kinder-DIPS was administered by a doctoral student and an advanced graduate clinical psychology student who had been specifically trained in the administration of this interview.

Children with social phobia were excluded if SP was not the primary diagnosis or if they had a co-morbid DSM-IV diagnosis of major depression, dysthymia or autism. In the SP group, 15 (36.6\%) of the 41 children fulfilled the DSM-IV criteria of a secondary disorder. The following co-morbid conditions were diagnosed: attention deficit hyperactivity disorder $(n=5)$, specific phobia $(n=6)$, oppositional-defiant disorder $(n=1)$, obsessive-compulsive disorder $(n=1)$, and enuresis $(n=2)$. Exclusion criteria for all children included medical conditions that might affect the investigated physiological systems (e.g. asthma). If there was a use of medication that could alter physiological responses (e.g. methylphenidate), children were excluded from physiological analyses. Children were asked to refrain from eating and drinking (except water) for at least $30 \mathrm{~min}$ before the beginning of the laboratory session since food in saliva is known to alter cortisol values. After the diagnostic interview, children and parents filled out several questionnaires on demographics and psychopathology.

\section{Procedure}

Following the diagnostic interview session, a second laboratory session was scheduled. To hold circadian influences on the measures constant all laboratory sessions took place between 3 and $5 \mathrm{pm}$.

After arrival at the laboratory, parents left the laboratory and children could adapt to the experimental situation for 15 min before being seated in a comfortable armchair in a sound attenuated, temperature controlled room. Ten minutes after the electrode hook-up the 5-min baseline period began, so overall children had a 30 -min acclimatization phase. Figure 1 illustrates the procedure and the timing of measurements. The baseline was followed by a 15-min preparation phase which included listening to the beginning of a story, preparing the continuation of the story and completing ratings. Then, $50 \mathrm{~min}$ after the beginning of the laboratory session, the speech element of the TSST-C commenced with the instructions for the child to continue telling the story for $5 \mathrm{~min}$ in front of a committee and a video camera "as exciting as possible" and to "perform better than other children their age". Subsequently the two unknown observers came in, the experimenter left the room and the $5 \mathrm{~min}$ speech began. Whenever children stopped talking, one of the observers prompted them to continue ("There is time left, please continue telling the story") while maintaining a neutral facial expression. At the end of the speech task (termed TSST-C speech) the experimenter returned for the 5-min mental arithmetic task (termed 
Fig. 1 Phases of the TSST-C along with measurement time points and HR-averaging segments; Anx = anxiety rating; $\mathrm{S}=$ saliva sample

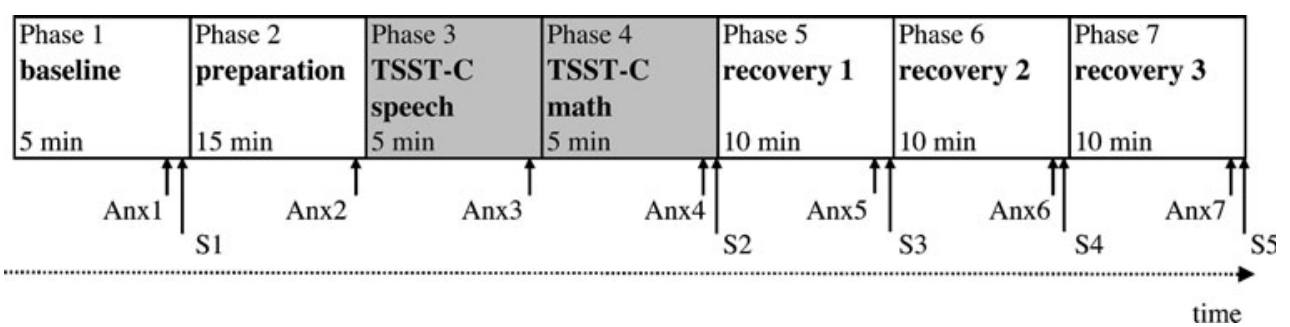

TSST-C math). Children were instructed to serially subtract the number 7 from 758 (for children 9 through 11 years) or the number 13 from 1023 (12 years) as fast and as accurately as possible. Because the original TSST-C does not include a mental arithmetic task for 8-year-old children, we added the task "98-3" for subjects aged 8. Slightly deviating from the original TSST-C-protocol and in order to equate groups on error feedback due to potential differences in math ability, four standardized error feedbacks were given every minute irrespective of actual mistakes ("Stop, there was an error in your last few steps, please start over again"). The TSST-C was followed by three 10-min resting periods (termed recovery 1 , recovery 2 and recovery 3 ) in which children watched pictures of landscapes on the computer. After this, electrodes were removed and the children were debriefed.

\section{Psychometric measures}

The Kinder-DIPS (Schneider et al. 2009) is a modified and extended version of the Anxiety Disorders Interview Schedule for children (ADIS-C; Silverman and Nelles 1988). It is a well-validated structured psychiatric interview which classifies frequent mental disorders in children and adolescents from 6 to 18 years according to the DSM-IV. It consists of two separate interviews for child and parent. To make a final DSM-IV diagnosis, symptom criteria as well as impairment ratings according to the parent, the child and to both interviewers are taken into account. This interview shows good reliability and validity (Schneider et al. 1995).

The Social Phobia and Anxiety Inventory for Children (SPAI-C; Beidel et al. 1995) is a self-report questionnaire, which is widely used to assess social anxiety in childhood. It consists of 26 items measuring different physical, cognitive and avoidant symptoms of social anxiety according to the criteria of the DSM-IV (APA, 1994). A total score between 0 and 52 is calculated. The German SPAI-C (Melfsen et al. 2001) has high internal consistency $(\alpha=$ $0.95)$ and high test-retest reliability $(r=0.84)$. The SPAI-C discriminates well between socially and non-socially phobic individuals with a cut-off score at 18 .

The Child Depression Inventory (CDI) (Kovacs 1985) consists of 26 items and assesses the cognitive, affective, and behavioral symptoms of depression in childhood. Total scores range from 0 to 52 . The German CDI (Stiensmeier-
Pelster et al. 2000) demonstrated good internal consistency $(\alpha=0.84)$ and satisfying test-retest reliability $(r=0.76)$ and distinguishes children with major depression from nondepressed children with a recommended cut-off score at 18 .

During the same session, parents completed several questionnaires. For the present study, we report data from the German version of the Child Behavior Checklist (CBCL; Achenbach 1991). The CBCL completed by the parents consists of 113 items and two broad scales, representing externalizing behavior problems and internalizing behavior problems. This questionnaire is widely used to assess early social adjustment and has adequate reliability and validity.

All questionnaires showed good internal consistencies in the present sample ( $\alpha=0.85$ through $\alpha=0.96)$.

\section{Subjective anxiety}

Children rated their anxiety at seven time points during the experimental procedure (see also Fig. 1) using developmentally appropriate 11-point Likert scales taken out of the Scales for Iconic Self-Assessment of Anxiety in Children (ISAAC; Schneider et al. 2005). Retrospective anxiety ratings were obtained after baseline, preparation, TSST-C speech and TSST-C math, recovery 1 , recovery 2 , recovery 3 , each referring to the maximum anxiety in the last period.

\section{Heart rate}

From an Einthoven lead 1 setup, the electrocardiogram (ECG) was sampled at $400 \mathrm{~Hz}$, streamed to disk and displayed on a PC monitor using the Varioport system (Becker Meditec, Karlsruhe, Germany). Data inspection and artefact rejection was performed offline using ANSLAB (Wilhelm and Peyk 2005). For the ECG, cardiac interbeat interval (IBI), calculated as the interval in milliseconds between successive R-waves, was extracted. The mean IBI was obtained for each of the seven experimental phases. For illustrative purposes the IBI was converted to HR (in beats per minute [bpm]) for tables and figures but all statistical analyses where based on IBI values (Quigley and Berntson 1996).

Salivary biomarkers: sAA and cortisol

A total of five saliva samples were obtained during the laboratory session to analyze salivary cortisol and sAA 
concentrations. As can be seen from Fig. 1, saliva samples were collected together with the anxiety ratings (Anx), however, not after Anx2 and Anx3. Due to slow response, salivary cortisol increases can be observed only 15-20 min after the start of an effective stressor (Kirschbaum and Hellhammer 1994). Saliva samples were obtained by having children chew on a Salivette (SarstedtNumbrecht, Germany) for $1 \mathrm{~min}$. Then Salivette collection devices were stored at $-20^{\circ} \mathrm{C}$ until assayed. At two time points during data acquisition every study site sent the saliva samples collected until then to the laboratory of Professor Kirschbaum at the Technical University of Dresden, Germany, where the samples were analyzed collectively. For sAA analyses a quantitative enzyme kinetic method was used as described in detail elsewhere (van Stegeren et al. 2006). Salivary cortisol levels were measured by use of commercial immunoassay with chemiluminescence detection (IBLHamburg, Hamburg, Germany). Intra- and inter-assay precision expressed as percent coefficient of variation was below $10 \%$ for both cortisol and sAA assay.

\section{Statistical analyses}

Outliers (z-score $>+-3$ based on group mean) and missing values were excluded from the respective analyses, leading to varying degrees of freedom for the dependent variables. ${ }^{2}$

Separate mixed ANOVAs with the repeated measures factor Phase (baseline, preparation, TSST-C speech, TSST-C math, recovery 1 , recovery 2 , recovery 3 for anxiety and HR; baseline, TSST-C, recovery 1 , recovery 2 , recovery 3 for cortisol and sAA) and the between participants factor Group (HC, SP) were calculated for each of the four primary measures (anxiety ratings, HR, salivary cortisol, sAA). Significant Group X Phase interactions were decomposed using separate Repeated Measures ANOVAs for reactivity (increase from baseline to first stress task: baseline, preparation, TSST-C speech) and recovery (second stress task to post-experimental baseline: TSST-C math, recovery 1 , recovery 2 , recovery 3 ) as suggested by previous research (e.g., Kamarck and Lovallo 2003; Linden et al. 2003; Seeman and Robbins 1994). Independent sample t-tests were used to decompose Group effects. As effect-size measures partial $\eta^{2}$ was reported for ANOVA results and Cohen's $d$ for t-tests. The significance level for all analyses was set at $\alpha=0.05$. All statistical tests were performed two-tailed and $d f$ s were Greenhouse-Geisseradjusted. For reasons of brevity, Phase effects were only reported when interacting with Group.

\footnotetext{
$\overline{2}$ Including outliers into statistical analyses did not change the results. Outliers: HR: 1 participant (HC), salivary cortisol: 3 participants (SP), sAA: 3 participants (1 SP, $2 \mathrm{HC}$ ); Missing values due to artefacts or technical errors: HR: 3 participants $(2 \mathrm{SP}, 1 \mathrm{HC})$, salivary cortisol: 1 participants (SP), sAA: 4 participants (1 SP, $3 \mathrm{HC})$.
}

\section{Results}

\section{Participant characteristics}

Table 1 shows demographic and psychometric measures for the two groups. The groups did not differ in terms of gender, age, and school level and there was no difference between the two sites in participant demographics (all $p \mathrm{~s}>$ 0.418 ). In accordance with the diagnostic categorization, the SP children had significantly higher scores on all symptom measures compared with the HC children. The mean SPAI-C score in the SP group was above the clinical cut-off score of 18 .

\section{Anxiety ratings}

Figure 2a shows the mean absolute levels of subjective anxiety ratings for both groups in each experimental phase. To investigate differences between the two groups on subjective anxiety, an omnibus $2 \times 7$ Group (SP, HC) X Phase (baseline, preparation, TSST-C speech, TSST-C math, recovery 1-3) ANOVA was computed. Significant main effects for Phase, $F(6,474)=133.9, p<0.001, \eta^{2}=0.629$, and Group, $F(1,79)=$ $49.0, p<0.001, \eta^{2}=0.383$, were modulated by a Phase $\mathrm{X}$ Group interaction, $F(6,474)=9.28, p<0.001, \eta^{2}=0.105$. Following up on this interaction we computed separate ANOVAs for reactivity and recovery. The $2 \times 3$ Group (SP, HC) X Phase (baseline, preparation, TSST-C speech) reactivity ANOVA revealed a main effect for Group, $F(1,79)=54.2, p<0.001, \eta^{2}=0.407$, and a Group X Phase interaction, $F(2,158)=7.70, p=0.001, \eta^{2}=0.089$ (linear trend, $\left.p<0.001, \eta^{2}=0.121\right)$, indicating a steeper linear anxiety increase in the SP group, compared to HC. The $2 \times 4$ Group (SP, HC) X Phase (TSST-C math, recovery1-3) recovery ANOVA yielded a main effect for Group, $F(1,79)=29.4, p<$ $0.001, \eta^{2}=0.271$, and a Group $X$ Phase interaction, $F(3,237)=$ 5.74, $p=0.009, \eta^{2}=0.068$, illustrating a faster linear decline in the SP group (linear trend, $p=0.004, \eta^{2}=0.099$ ). Among the two groups, the SP group had higher anxiety ratings at all time points (all $t \mathrm{~s}>3.53$, all $p \mathrm{~s}<0.01$ ). ${ }^{3}$

\section{Heart rate}

Mean absolute levels of HR for both groups in each phase are presented in Fig. 2b. A $2 \times 7$ Group X Phase ANOVA yielded significant main effects for Phase, $F(6,450)=101.3, p<$ $0.001, \eta^{2}=0.575$, and Group, $F(1,75)=4.54 ; p=0.036, \eta^{2}=$ 0.057 , in addition to a Group X Phase interaction, $F(6,450)=$

\footnotetext{
${ }^{3}$ ANCOVAs evaluated the effect of the covariates study site, gender, and presence of comorbidity on the dependent variables. In no case did inclusion of these covariates alter the interpretation of the results.
} 
Table 1 Participant characteristics

\begin{tabular}{llll}
\hline & $\begin{array}{l}\text { Social } \\
\text { phobia } \\
\text { M (SD) }\end{array}$ & $\begin{array}{l}\text { Healthy } \\
\text { controls } \\
\text { M (SD) }\end{array}$ & Statistics \\
\hline $\begin{array}{llll}\text { N in sample } \\
\quad \text { Site 1/Site 2) }\end{array}$ & $41(22 / 19)$ & $40(20 / 20)$ & \\
$\begin{array}{l}\text { Age (years) } \\
\text { Gender (male/female) }\end{array}$ & $10.1(1.39)$ & $10.0(1.12)$ & $t(79)=0.17$ \\
$\begin{array}{l}\text { School } \\
\text { (primary/secondary) }\end{array}$ & $22 / 19$ & $20 / 20$ & $\chi^{2}(1)=0.01$ \\
$\begin{array}{l}\text { SPAI-C } \\
\text { CDI }\end{array}$ & $20.0(8.09)$ & $3.94(2.96)$ & $t(78)=11.89 * * *$ \\
CBCL externalizing & $8.48(7.74)$ & $3.79(3.32)$ & $t(77)=3.52^{* * *}$ \\
$\quad$ score & $16.99(7.70)$ & $2.86(2.83)$ & $t(77)=10.9 * * *$ \\
CBCL Internalizing & & & \\
$\quad$ score & $35.3(16.4)$ & $8.99(5.71)$ & $t(77)=9.56^{* * *}$ \\
CBCL total score & & & \\
\hline
\end{tabular}

$M$ mean, $S D$ standard deviation, SPAI-C Social Phobia and Anxiety Inventory for Children, $C D I$ Child Depression Inventory, $C B C L$ Child Behavior Checklist; ${ }^{* *} \mathrm{p}<0.01,{ }^{* * *} \mathrm{p}<0.001$

$4.05 ; p=0.011 ; \eta^{2}=0.051$. The follow-up $2 \times 3$ Group by Phase reactivity ANOVA yielded a significant main effect for Group, $F(1,75)=5.31, p=0.024, \eta^{2}=0.066$, and a significant Group X Phase interaction, $F(2,150)=3.96, p=0.030, \eta^{2}=$ 0.05 , pointing to a blunted HR response in the SP group (linear trend, $p=0.028 ; \eta^{2}=0.063$ ). The $2 \times 4$ recovery ANOVA yielded a trend level main effect for Group, $F(1,75)=3.759, p=0.056, \eta^{2}=0.048$, and a significant Group $\mathrm{X}$ Phase interaction, $F(3,225)=5.88, p=0.009, \eta^{2}=0.073$, revealing a slower decrease of the HR in the SP group compared to the $\mathrm{HC}$ group (linear trend, $p=0.009$; $\eta^{2}=$ 0.088). Among the two groups, the SP group reached higher HR levels at all time points except TSST-C speech and TSST$\mathrm{C}$ math $\left(t_{\mathrm{s}}>-2.118, p \mathrm{~s}<0.038\right)$.

\section{Salivary $\alpha$ lpha-amylase}

A $2 \times 5$ Group (SP, HC) X Phase (baseline, TSST-C, recovery 1-3) ANOVA with repeated measures on the Phase factor did not yield a significant main effect for Group $F(1,72)=1.96, p=0.166$, and no significant Group $\mathrm{X}$ Phase interaction, $F(4,288)=1.85, p=0.146$, demonstrating that groups did show similar responding patterns to the TSST-C (Fig. 2c). ${ }^{4}$ On a descriptive level, the SP children had even lower sAA levels than the controls.

\footnotetext{
${ }^{4}$ Additional group comparisons concerning values for area under the curve with respect to ground (AUCg) and area under the curve with respect to increase (AUCi; Pruessner et al. 2003) did not show significant differences between SPs and HCs $(p s>0.125)$.
}

Salivary cortisol

In a $2 \times 5$ Group (SP, HC) X Phase ANOVA with repeated measures on the Phase factor, the main effect for Group did not reach significance, $F(1,75)=0.880, p=0.351$, nor did the Group X Phase interaction, $F(4,300)=1.45, p=0.238$, indicating that the groups showed similar responding patterns to the TSST-C (Fig. 2d). Similarly to sAA, on a descriptive level, the SP children had an even lower cortisol response compared to the controls. ${ }^{5}$

\section{Discussion}

To our knowledge, this is the first study to provide a multilevel analysis of subjective, autonomic and endocrine reactivity to a psychosocial stressor in SP children. The results can be summarized as follows. In addition to a generally elevated subjective anxiety level during the whole task, SP children showed a stronger reactivity (steeper increase of anxiety) and a faster recovery (steeper decrease of anxiety) compared to controls. Similar to subjective anxiety, HR was also chronically elevated throughout the task in SP children, even though they showed a blunted reactivity (slower increase in HR levels) and recovery (slower decrease of HR levels) compared to controls. No differences between the SP and HC groups were obtained for sAA and salivary cortisol. In the following, we discuss our findings separately for subjective anxiety, autonomic arousal and endocrine stress responses.

\section{Subjective anxiety}

Stronger anxiety reactivity corresponds well with prior findings, demonstrating higher reactivity in anxiety in SP children compared to controls during social-evaluative tasks (Beidel 1991; Spence et al. 1999; van West et al. 2008). Subjective anxiety hyperreactivity in SP children also speaks for a successful symptom provocation by the TSST-C and points to a specific sensitivity of these children to social-evaluative contexts, which is well in line with current nosology and theory. Enhanced reactivity to the stressor in the SP children was accompanied by a faster recovery. However, overall anxiety remained elevated in the SP children throughout the task, which could be due to either reporting biases or to chronically elevated subjective anxiety in a variety of social contexts.

\footnotetext{
${ }_{5}^{5}$ Additional group comparisons concerning values for area under the curve with respect to ground (AUCg) and area under the curve with respect to increase (AUCi; Pruessner et al. 2003) did not show significant differences between SPs and HCs $(p s>0.722)$.
} 
Fig. 2 Means (and standard errors) of self-reported and physiological responses to the TSST-C in SP children and HC. Panels depict anxiety ratings $\mathbf{a}$, heart rate $\mathbf{b}$, salivary alphaamylase (sAA) $\mathbf{c}$ and salivary cortisol d
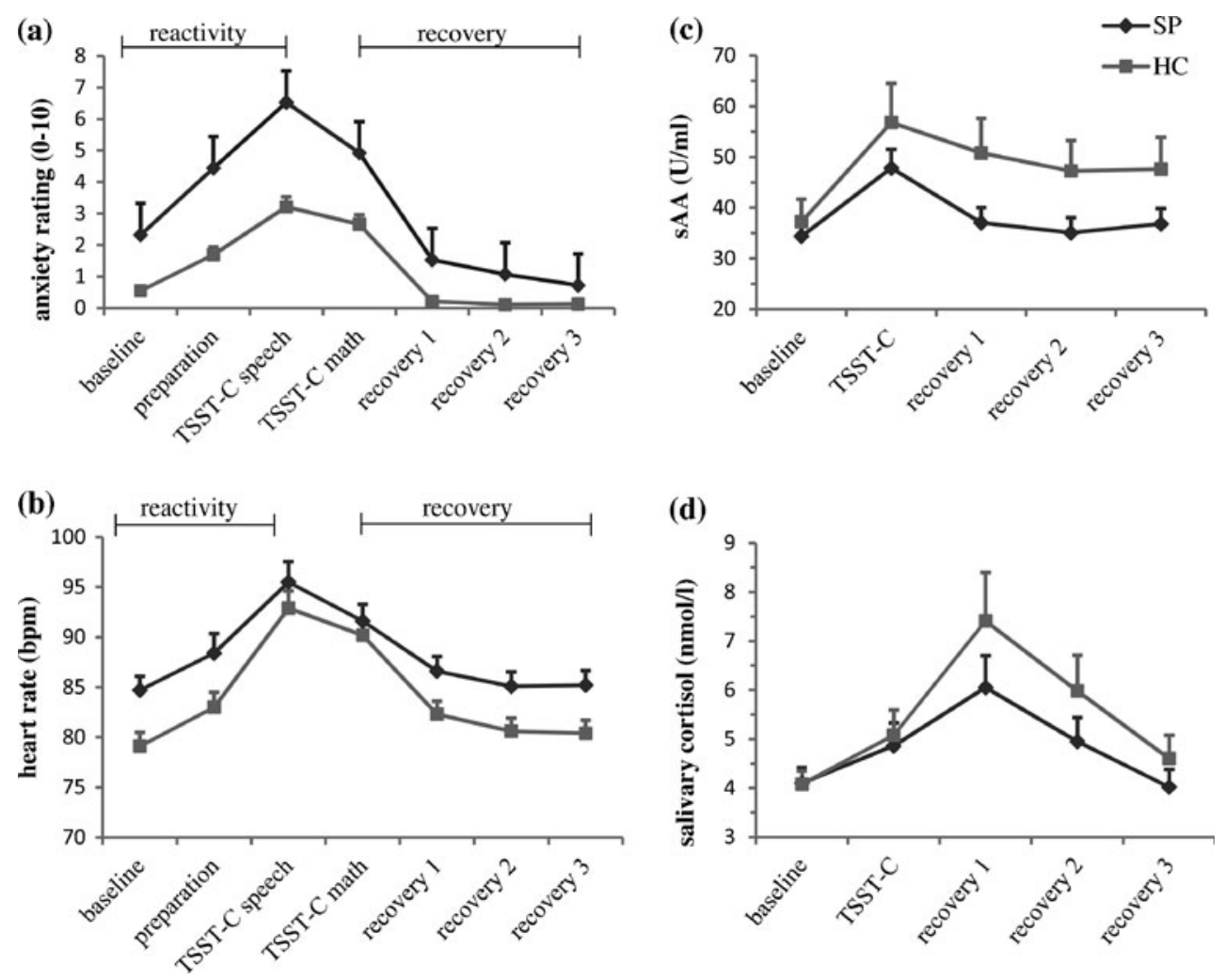

\section{Heart rate}

Consistent with elevated chronic subjective anxiety, HR was also found to be higher in SP children throughout the experiment. However, contrary to the pattern of subjective anxiety, we found a blunted HR reactivity (slower increase) to the TSST-C and a slowed recovery (slower decrease) in SP children. Previous studies did also not find higher HR reactivity in SP children and adolescents compared to HC (Anderson and Hope 2009; Beidel 1991). In addition, our results correspond with findings of elevated HR levels during rest and stress in samples of mixed anxiety disordered children and adolescents (Monk et al., 2001) and behaviorally inhibited children (Kagan et al. 1988).

The presence of subjective hyperreactivity to stress, unaltered acute HR reactivity, but chronically elevated HR at baseline as well as during recovery can be interpreted in two different ways. First, the blunted HR reactivity in SP children could be an effect of physiological ceiling effects. Due to baseline dependencies of measures like HR, further HR increases from an elevated baseline might have been constrained by physiological counter-regulatory effects. Determining the presence of physiological ceiling effects would require an more direct assessment of both parasympathetic and sympathetic ANS divisions (Berntson et al. 1993). We have recently reported on a reduced autonomic flexibility in children with SP as a potential explanation for reduced stress reactivity in the presence of tonic hyperarousal (Schmitz et al. 2011). ${ }^{6}$

A second potential explanation of blunted acute HR reactivity on the background of chronically elevated HR in SP children is that they might not be hyperreactive to social-evaluative stress specifically, but that they are instead chronically over-aroused in a variety of unspecified social situations. It is noteworthy that we found elevated HR levels not only at baseline, where it could be explained by elevated anticipatory anxiety, but also during recovery, and therefore in the absence of anticipatory anxiety (children knew that they had completed the task and no more challenges would be forthcoming). Constantly receiving afferent feedback about elevated physiological arousal, the SP children might have started worrying about the visibility of signs of arousal and anxiety as shown to be the case for adults with SP (Gerlach et al. 2004). In fact, a recent study from our lab indicates that socially anxious children worry more about their arousal symptoms than non-anxious control children even in the absence of group differences in HR and HR perception (as measured in a heart-beat counting task) (Schmitz et al., in revision; see also Anderson and Hope 2009). Importantly, in the long run, chronic physiological hyperarousal could lead to attentional biases similar to the ones described by Clark and Wells

\footnotetext{
${ }^{6}$ Please note that analysis of covariance does not provide a solution here (Miller and Chapman 2001).
} 
(1995) of directing attention inward toward bodily symptoms instead of outward toward the socially demanding situations (Spurr and Stopa 2002). Ultimately, the question of how prevalent phases of elevated HR levels are in SP children has to be studied in ambulatory monitoring studies, which are currently lacking in this population. Should such studies confirm chronically elevated HR levels in a wide array of social situations, significant implications for cardiovascular health would have to be considered (Thayer et al. 2010).

\section{Salivary alpha-amylase}

We did not find any differences between the SP and HC children in terms of sAA reactivity. Both groups showed a significant increase in SAA in response to the TSST-C, which shows its sensitivity to social stress (Nater and Rohleder 2009). However, sAA reactivity did not reflect the group differences found on HR and subjective anxiety. This result of an absence of group differences in sAA reactivity to the TSST-C found in this present study seems inconsistent with van Veen et al. (2008) who reported on elevated sAA levels in adults with SP when compared to HCs. These differences might be due to the lack of a stressor in van Veen's study. Moreover, it has to be considered that there might be differences between children and adults with regard to sAA reactivity (Strahler et al. 2010; Yim et al. 2010). Therefore, more research is needed on the role of sAA in childhood SP as well as on how it relates to HR levels and subjective anxiety in general.

\section{Salivary cortisol}

Contrary to our assumption, we did not find any differences between SP and HC children in salivary cortisol. This result is consistent with studies with adolescents and adults reporting similar cortisol reactivity in $\mathrm{SP}$ and $\mathrm{HC}$ groups (Beaton et al. 2006; Heiser et al. 2009). However, it is inconsistent with results of van West et al. (2008), who reported higher cortisol reactivity in children with SP compared to $\mathrm{HC}$ in response to social stress. The present study and the study of van West et al. (2008) differ in stressor length (twice as long in the present study), age group (present study 8-12, van West et al. 6-12 years), and sample size (present study 40/group, van West et al. 25/ group). Cortisol reactivity is influenced by intensity and length of the stressor as well as by participant age (Dickerson and Kemeny 2004; Kudielka et al. 2004), which may account for some of the differences. Keeping these discrepancies in mind, our results suggest that the symptoms of childhood SP may not be generalized to the HPA-axis-based neuroendocrine stress system. The question which factors may specifically contribute to a closer covariation of both dimensions in SP children, such as participant age or nature of the social situation, needs further investigation.

\section{Limitations}

Several limitations must be acknowledged. First, our study assessed subjective, autonomic and endocrine reactivity to social stress during a highly structured social-evaluative task. Even though situations such as the ones faced in our speech and math task are quite common in the everyday life, future research should expand tasks to encompass a wider variety of externally valid situations such as, e.g., informal conversation with peers.

Second, we only investigated children between 8 and 12 years. This was done to capture the earliest manifestations of the disorder which develop during this time (Beidel et al. 1999). Therefore we cannot draw conclusions about SP in adolescence. Future studies should compare subjective, autonomic, and endocrine reactivity to social stress among different age groups of youth with SP, because previous results indicate that manifestation of SP differs in childhood and adolescence (e.g. Alfano et al. 2006; Rao et al. 2007).

Third, it is not clear how representative our sample was. It is possible that mainly families with higher education and higher social economic status responded to our advertisements. Further concerns related our sample could be that only Caucasian children participated in our study because there is empirical evidence that the expression of social anxiety differs between ethnical groups (Asnaani et al. 2010). Thus, future research comparing subjective, autonomic, and endocrine reactivity of SP in different ethical groups is needed.

Finally, as mentioned above, ambulatory research should examine whether HR is elevated tonically or whether this is an epiphenomena of laboratory research (Wilhelm et al. 2006).

Conclusions and future directions

The present results demonstrate the expected stronger subjective anxiety reactivity in SP children compared to HC children. In addition, SP children are characterized by chronically elevated HR levels in conjunction with a blunted HR response to the TSST-C. Future studies should target the coupling between subjective anxiety and physiological stress symptoms to understand their interaction and potential feedback loops, for example by acquiring anxiety and arousal ratings at high temporal resolution in addition to continuous multi-channel physiological assessment (Burkhardt et al. 2010). Finally, further research is needed to provide clarification if current therapeutic approaches for 
children with social phobia target physiological hyperarousal and dysfunctional interpretation of bodily symptoms effectively.

Acknowledgements This research was funded by the German Research Foundation (DFG; Grant TU 78/5-1; HE 3342/4-1).

\section{References}

Achenbach, T. M. (1991). Manual for the child behavior checklist 418 and 1991 profile. Burlington: University of Vermont, Department of Psychiatry.

Alfano, C. A., Beidel, D. C., \& Turner, S. M. (2006). Cognitive correlates of social phobia among children and adolescents. Journal of Abnormal Child Psychology, 34(2), 189-201.

American Psychiatric Association. (1994). Diagnostic and statistical manual of mental disorders (4th ed.). Washington: American Psychiatric Association.

Anderson, E. R., \& Hope, D. A. (2009). The relationship among social phobia, objective and perceived physiological reactivity, and anxiety sensitivity in an adolescent population. Journal of Anxiety Disorders, 23(1), 18-26.

Asnaani, A., Richey, J. A., Dimaite, R., Hinton, D. E., \& Hofmann, S. G. (2010). A cross-ethnic comparison of lifetime prevalence rates of anxiety disorders. The Journal of Nervous and Mental Disease, 198(8), 551-555.

Axelrod, J., \& Reisine, T. D. (1984). Stress hormones: their interaction and regulation. Science, 224, 452-459.

Beaton, E. A., Schmidt, L. A., Ashbaugh, A. R., Santesso, D. L., Antony, M. M., McCabe, R. E., et al. (2006). Low salivary cortisol levels among socially anxious young adults: preliminary evidence from a selected and a non-selected sample. Personality and Individual Differences, 41(7), 1217-1228.

Beidel, D. C. (1991). Social phobia and overanxious disorder in school-age children. Journal of the American Academy of Child and Adolescent Psychiatry, 30(4), 545-552.

Beidel, D. C., Turner, S. M., \& Morris, T. L. (1995). A new inventory to assess childhood social anxiety and phobia: the social phobia and anxiety inventory for children. Psychological Assessment, 7, 73-79.

Beidel, D. C., Turner, S. M., \& Morris, T. L. (1999). Psychopathology of childhood social phobia. Journal of the American Academy of Child and Adolescent Psychiatry, 38(6), 643-650.

Berntson, G. G., Cacioppo, J. T., \& Quigley, K. S. (1993). Cardiac psychophysiology and autonomic space in humans: empirical perspectives and conceptual implications. Psychological Bulletin, 114, 296-322.

Berntson, G. G., Cacioppo, J. T., Binkley, P. F., Uchino, B. N., Quigley, K. S., \& Fieldstone, A. (1994). Autonomic cardiac control. III. Psychological stress and cardiac response in autonomic space as revealed by pharmacological blockades. Psychophysiology, 31 (6), 599-608.

Burkhardt, S. C., Wilhelm, F. H., Meuret, A. E., Blechert, J., \& Roth, W. T. (2010). Temporal stability and coherence of anxiety, dyspnea, and physiological variables in panic disorder. Biological Psychology, 85, 226-232.

Buske-Kirschbaum, A., Jobst, S., Wustmans, A., Kirschbaum, C., Rauh, W., \& Hellhammer, D. (1997). Attenuated free cortisol response to psychosocial stress in children with atopic dermatitis. Psychosomatic Medicine, 59(4), 419-426.

Clark, D. M., \& Wells, A. (1995). A cognitive model of social phobia. In R. G. Heimberg (Ed.), Social phobia-diagnosis, assessment, and treatment (pp. 69-93). New York: Guilford.
Dickerson, S. S., \& Kemeny, M. E. (2004). Acute stressors and cortisol responses: a theoretical integration and synthesis of laboratory research. Psychological Bulletin, 130(3), 355-391.

Gerlach, A. L., Mourlane, D., \& Rist, F. (2004). Public and private heart rate feedback in social phobia: a manipulation of anxiety visibility. Cognitive Behaviour Therapy, 33(1), 36-45.

Grossman, P., Wilhelm, F. H., Kawachi, I., \& Sparrow, D. (2001). Gender differences in psychophysiological responses to speech stress among older social phobics: congruence and incongruence between self-evaluative and cardiovascular reactions. Psychosomatic Medicine, 63(5), 765-777.

Heiser, N. A., Turner, S. M., Beidel, D. C., \& Roberson-Nay, R. (2009). Differentiating social phobia from shyness. Journal of Anxiety Disorders, 23(4), 469-476.

Hofmann, S. G., Newman, M. G., Ehlers, A., \& Roth, W. T. (1995). Psychophysiological differences between subgroups of social phobia. Journal of Abnormal Psychology, 104(1), 224-231.

Kagan, J., Reznick, J. S., \& Snidman, N. (1988). Biological bases of childhood shyness. Science, 240(4849), 167-171.

Kamarck, T. W., \& Lovallo, W. R. (2003). Cardiovascular reactivity to psychological challenge: conceptual and measurement considerations. Psychosomatic Medicine, 65(1), 9-21.

Kessler, R. C. (2003). The impairments caused by social phobia in the general population: implications for intervention. Acta Psychiatrica Scandinavica. Supplementum, 108(417), 19-27.

Kirschbaum, C., \& Hellhammer, D. H. (1994). Salivary cortisol in psychoneuroendocrine research: recent developments and applications. Psychoneuroendocrinology, 19(4), 313-333.

Kirschbaum, C., Pirke, K. M., \& Hellhammer, D. H. (1993). The 'Trier Social Stress Test'-a tool for investigating psychobiological stress responses in a laboratory setting. Neuropsychobiology, 28(1-2), 76-81.

Kovacs, M. (1985). The Children's Depression Inventory (CDI). Psychopharmacology Bulletin, 21(4), 995-999.

Kudielka, B. M., Buske-Kirschbaum, A., Hellhammer, D. H., \& Kirschbaum, C. (2004). Differential heart rate reactivity and recovery after psychosocial stress (TSST) in healthy children, younger adults, and elderly adults: the impact of age and gender. International Journal of Behavioral Medicine, 11(2), 116-121.

Linden, W., Gerin, W., \& Davidson, K. (2003). Cardiovascular reactivity: status quo and a research agenda for the new millennium. Psychosomatic Medicine, 65(1), 5-8.

Mauss, I. B., Wilhelm, F. H., \& Gross, J. J. (2003). Autonomic recovery and habituation in social anxiety. Psychophysiology, 40 (4), 648-653.

Melfsen, S., Florin, I., \& Warnke, A. (2001). Sozialphobie- und angstinventar für Kinder (SPAIK). Manual. Göttingen: Hogrefe.

Miller, G. A., \& Chapman, J. P. (2001). Misunderstanding analysis of covariance. Journal of Abnormal Psychology, 110(1), 40-48.

Monk, C., Kovelenko, P., Ellman, L. M., Sloan, R. P., Bagiella, E., Gorman, J. M., et al. (2001). Enhanced stress reactivity in paediatric anxiety disorders: implications for future cardiovascular health. International Journal of Neuropsychopharmacology, 4 (2), 199-206.

Nater, U. M., \& Rohleder, N. (2009). Salivary alpha-amylase as a noninvasive biomarker for the sympathetic nervous system: current state of research. Psychoneuroendocrinology, 34(4), 486-496.

Nater, U. M., Rohleder, N., Gaab, J., Berger, S., Jud, A., Kirschbaum, C., et al. (2005). Human salivary alpha-amylase reactivity in a psychosocial stress paradigm. International Journal of Psychophysiology, 55(3), 333-342.

Ollendick, T. H., \& Hirshfeld-Becker, D. R. (2002). The developmental psychopathology of social anxiety disorder. Biological Psychiatry, 51(1), 44-58.

Pruessner, J. C., Kirschbaum, C., Meinlschmid, G., \& Hellhammer, D. H. (2003). Two formulas for computation of the area under the 
curve represent measures of total hormone concentration versus time-dependent change. Psychoneuroendocrinology, 28(7), 916931.

Quigley, K. S., \& Berntson, G. G. (1996). Autonomic interactions and chronotropic control of the heart: heart period versus heart rate. Psychophysiology, 33(5), 605-611.

Rao, P. A., Beidel, D. C., Turner, S. M., Ammerman, R. T., Crosby, L. E., \& Sallee, F. R. (2007). Social anxiety disorder in childhood and adolescence: descriptive psychopathology. Behaviour Research and Therapy, 45(6), 1181-1191.

Rapee, R. M., \& Heimberg, R. G. (1997). A cognitive-behavioral model of anxiety in social phobia. Behaviour Research and Therapy, 35(8), 741-756.

Roelofs, K., van Peer, J., Berretty, E., Jong, P., Spinhoven, P., \& Elzinga, B. M. (2009). Hypothalamus-pituitary-adrenal axis hyperresponsiveness is associated with increased social avoidance behavior in social phobia. Biological Psychiatry, 65(4), 336-343.

Ruscio, A. M., Brown, T. A., Chiu, W. T., Sareen, J., Stein, M. B., \& Kessler, R. C. (2008). Social fears and social phobia in the USA: results from the National Comorbidity Survey Replication. Psychological Medicine, 38(1), 15-28.

Schmitz, J., Kramer, M., Tuschen-Caffier, B., Heinrichs, N., \& Blechert, J. (2011). Restricted autonomic flexibility in children with social phobia. J Child Psychol Psychiatry.

Schneider, S., Unnewehr, S., \& Margraf, J. (1995). Handbuch. In S. Unnewehr, S. Schneider, \& J. Margraf (Eds.), Diagnostisches Interview bei psychischen Störungen im Kindes- und Jugendalter. Kinder-DIPS. Berlin: Springer.

Schneider, S., Wilhelm, F. H., \& Martin, I. J. (2005). Scales for Iconic Self-Assessment of Anxiety in Children (ISAAC). Basel: University of Basel, Institute for Psychology.

Schneider, S., Unnewehr, S., \& Margraf, J. (Eds.). (2009). KinderDIPS: Diagnostisches Interview bei psychischen Störungen im Kindes- und Jugendalter (2nd ed.). Heidelberg: Springer.

Schneier, F. R., Johnson, J., Hornig, C. D., Liebowitz, M. R., \& Weissman, M. M. (1992). Social phobia. Comorbidity and morbidity in an epidemiologic sample. Archives of General Psychiatry, 49(4), 282-288.

Seeman, T. E., \& Robbins, R. J. (1994). Aging and hypothalamicpituitary-adrenal response to challenge in humans. Endocrine Reviews, 15(2), 233-260.
Silverman, W. K., \& Nelles, W. B. (1988). The anxiety disorders interview schedule for children. Journal of the American Academy of Child and Adolescent Psychiatry, 27(6), 772-778.

Spence, S. H., Donovan, C., \& Brechman-Toussaint, M. (1999). Social skills, social outcomes, and cognitive features of childhood social phobia. Journal of Abnormal Psychology, 108(2), 211-221.

Spurr, J. M., \& Stopa, L. (2002). Self-focused attention in social phobia and social anxiety. Clinical Psychology Review, 22(7), 947-975.

Stiensmeier-Pelster, J., Schürmann, M., \& Duda, K. (2000). Depressions-Inventar für Kinder und Jugendliche (2nd ed.). Göttingen: Hogrefe.

Strahler, J., Mueller, A., Rosenloecher, F., Kirschbaum, C., \& Rohleder, N. (2010). Salivary alpha-amylase stress reactivity across different age groups. Psychophysiology.

Thayer, J. F., Yamamoto, S. S., \& Brosschot, J. F. (2010). The relationship of autonomic imbalance, heart rate variability and cardiovascular disease risk factors. International Journal of Cardiology, 141(2), 122-131.

van Stegeren, A., Rohleder, N., Everaerd, W., \& Wolf, O. T. (2006). Salivary alpha amylase as marker for adrenergic activity during stress: effect of betablockade. Psychoneuroendocrinology, 31(1), 137-141.

van Veen, J. F., van Vliet, I. M., DeRijk, R. H., van Pelt, J., Mertens, B., \& Zitman, F. G. (2008). Elevated alpha-amylase but not cortisol in generalized social anxiety disorder. Psychoneuroendocrinology, 33(10), 1313-1321.

van West, D., Claes, S., Sulon, J., \& Deboutte, D. (2008). Hypothalamic-pituitary-adrenal reactivity in prepubertal children with social phobia. Journal of Affective Disorders, 111(2-3), 281290.

Wilhelm, F. H., \& Peyk, P. (2005). ANSLAB 4.0: Autonomic nervous system laboratory [software]. Available from http://www.sprweb. org.

Wilhelm, F. H., Pfaltz, M. C., Grossman, P., \& Roth, W. T. (2006). Distinguishing emotional from physical activation in ambulatory psychophysiological monitoring. Biomedical Sciences Instrumentation, 42, 458-463.

Yim, I. S., Granger, D. A., \& Quas, J. A. (2010). Children's and adults' salivary alpha-amylase responses to a laboratory stressor and to verbal recall of the stressor. Developmental Psychobiology, 52(6), 598-602. 